\title{
HABITABLE PLANETS ECLIPSING BROWN DWARFS: STRATEGIES FOR DETECTION AND CHARACTERIZATION
}

\author{
Adrian R. Belu ${ }^{1,2}$, Franck Selsis $^{1,2}$, Sean N. Raymond ${ }^{1,2}$, Enric Pallé ${ }^{3,4}$, Rachel Street $^{5}$, D. K. Sahu ${ }^{6}$, \\ Kaspar von Braun $^{7}$, Emeline Bolmont ${ }^{1,2}$, Pedro Figueira $^{8}$, G. C. Anupama ${ }^{6}$, and Ignasi Ribas ${ }^{9}$ \\ ${ }^{1}$ Université de Bordeaux, LAB, UMR 5804, F-33270, Floirac, France \\ ${ }^{2}$ CNRS, LAB, UMR 5804, F-33270 Floirac, France; belu@obs.u-bordeaux1.fr \\ ${ }^{3}$ Instituto de Astrofísica de Canarias, E-38205 La Laguna, Spain \\ ${ }^{4}$ Departamento de Astrofísica, Universidad de La Laguna, Av., Astrofísico Francisco Sánchez, s/n E-38206 La Laguna, Spain \\ ${ }^{5}$ Las Cumbres Observatory Global Telescope Network, 6740 Cortona Drive, Suite 102, Goleta, CA 93117, USA \\ ${ }^{6}$ Indian Institute of Astrophysics, Koramangala, Bangalore 560034, India \\ ${ }^{7}$ NASA Exoplanet Science Institute, California Institute of Technology, MC 100-22, Pasadena, CA 91125, USA \\ ${ }^{8}$ Centro de Astrofísica, Universidade do Porto, Rua das Estrelas, 4150-762 Porto, Portugal \\ ${ }^{9}$ Institut de Ciències de l'Espai (CSIC-IEEC), Campus UAB, Facultat de Ciències, Torre C5, parell, 2a pl., E-08193 Bellaterra, Spain \\ Received 2012 August 17; accepted 2013 January 8; published 2013 April 23
}

\begin{abstract}
Given the very close proximity of their habitable zones, brown dwarfs (BDs) represent high-value targets in the search for nearby transiting habitable planets that may be suitable for follow-up occultation spectroscopy. In this paper, we develop search strategies to find habitable planets transiting BDs depending on their maximum habitable orbital period $\left(P_{\mathrm{HZ} \text { out }}\right)$. Habitable planets with $P_{\mathrm{HZ} \text { out }}$ shorter than the useful duration of a night (e.g., 8-10 hr) can be screened with $100 \%$ completeness from a single location and in a single night (near-IR). More luminous BDs require continuous monitoring for longer duration, e.g., from space or from a longitude-distributed network (one test scheduling achieved three telescopes, 13.5 contiguous hours). Using a simulated survey of the 21 closest known BDs (within $7 \mathrm{pc}$ ) we find that the probability of detecting at least one transiting habitable planet is between $4.5_{-1.4}^{+5.6} \%$ and $56_{-13}^{+31} \%$, depending on our assumptions. We calculate that BDs within 5-10 pc are characterizable for potential biosignatures with a $6.5 \mathrm{~m}$ space telescope using $\sim 1 \%$ of a five-year mission's lifetime spread over a contiguous segment only one-fifth to one-tenth of this duration.
\end{abstract}

Key words: astrobiology - brown dwarfs - eclipses - infrared: planetary systems - instrumentation: spectrographs - solar neighborhood

\section{INTRODUCTION}

Together with in situ robotic exploration within our solar system, observations of terrestrial extra-solar planets' spectra is currently the most robust approach in the search for non-Earth life. The thermal emission from a habitable planet at $10 \mathrm{pc}$ is $\sim 1$ photons $\mathrm{s}^{-1} \mathrm{~m}^{-2} \mu \mathrm{m}^{-1}$; recording such a spectrum is within the capabilities of upcoming and even some existing space telescopes. Free-floating (rogue) planets, if yielding sufficient internal heat flow, could maintain habitable surface conditions if they also have adequate insulation, but this insulation would then limit the levels of photon emission, enabling characterization only for very nearby objects $(1000 \mathrm{AU}$ for the case of solid insulation; Abbot \& Switzer 2011). Therefore, the main approach until now has been to search for characterizable habitable planets around beacon primaries. These beacon primaries then become the dominant noise source when subsequently undertaking the characterization of the exoplanets.

Resolving the planet from the primary is therefore the first challenge. The spatial resolution of planets remains a technological challenge (Traub et al. 2007; Cockell et al. 2009). Fortuitously, transiting planets ${ }^{10}$ can be time-resolved from the brighter primary. Differential eclipse spectroscopy has enabled the identification of molecules in the atmospheres of giant planets close to solar-type stars (Tinetti et al. 2007; Grillmair et al. 2008; Swain et al. 2009; Stevenson et al. 2010;

10 The planet's orbit is passing in front of the star as seen from the telescope (primary eclipse).
Beaulieu et al. 2010). However, even the upcoming James Webb Space Telescope (JWST) will be able to detect biomarkers with this technique only up to $\sim 10 \mathrm{pc}$ and for primary dwarves approximately M5 and later (Beckwith 2008; Kaltenegger \& Traub 2009; Deming et al. 2009; Belu et al. 2011; Rauer et al. 2011; Pallé et al. 2011). Projects such as MEARTH (Charbonneau et al. 2008) are currently screening the solar neighborhood for such eclipsing planets. Yet the geometric transit likelihood and the local stellar population density and distribution do not guarantee the presence of even a single nearby transiting habitable planet suitable for characterization with JWST (Belu et al. 2011).

To help solve this scarcity problem we propose extending the search to primaries not yet considered by current surveys: brown dwarfs (BDs). Bright primary objects (primaries hereafter) are indeed required for transit spectroscopy, but as we quantify in this paper, occultation (secondary eclipse) spectroscopy (in emission) of habitable planets around a nearby $\mathrm{BD}$ is a favorable scenario. The intrinsic emission of a body that is at a given (habitable) equilibrium temperature is independent from the type of primary. On the contrary, the dimmer the primary, the less photon noise added to the planetary photons.

As said primaries are convenient "signposts," "lighthouses," or "beacons" for planets, but once the planets are found the primaries become a barrier to planetary characterization. So what is the dimmest lighthouse? Jupiter-sized objects colder than room temperature have been detected with the Wide-field Infrared Survey Explorer (WISE); see Cushing et al. 2011. BD primaries therefore should represent the optimal limit for the 


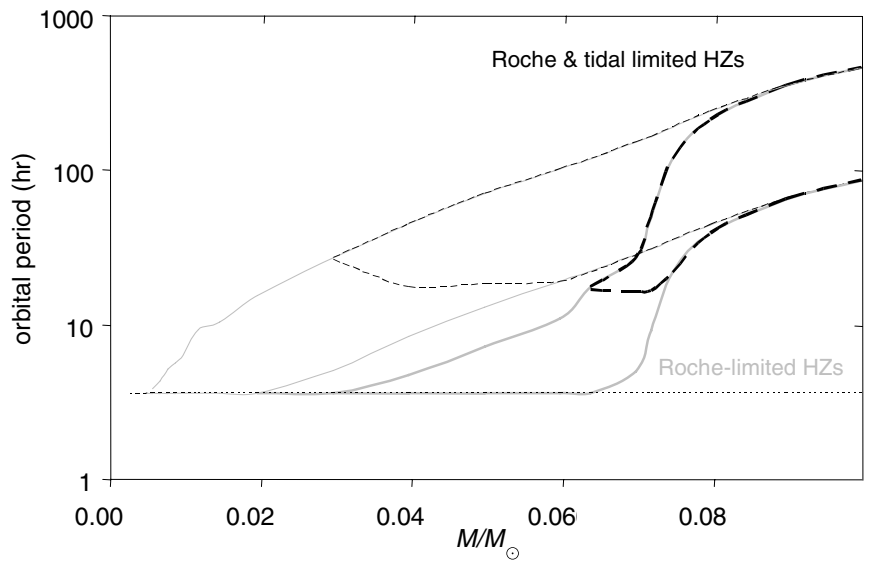

Figure 1. Orbital periods (in hours) of the effective habitable zone limits for 1 (thin lines) and 10 (thick lines) Gyr old BDs. The restricted black zones account for depletion of the inner HZs by tidal migration (see the text). The dotted line corresponds to the Roche period limit for the $10 M_{\oplus}, 1.8 R_{\oplus}$ super-Earth considered here: $3.6 \mathrm{hr}$.

"lighthouse" search paradigm for habitable exoplanets that can be time-resolved. ${ }^{11}$

But can planets form and remain habitable around BDs? There are significant differences between the potential habitability of planets around BDs and main-sequence stars. For instance, BDs cool in time and their habitable zone (HZ) moves inward such that a planet on a stationary orbit sees the HZ sweep by in a much shorter time interval than for stellar dwarfs (Caballero \& Rebolo 2002; Andreeschev \& Scalo 2004). However, there is no clear "Achilles' heel" that would rule out BDs as habitable planet hosts; this issue is discussed at length in Section 6. We thus continue with the assumption that BDs can indeed host habitable planets.

This paper is structured as follows. We first examine the observational characteristics of habitable planets eclipsing a BD (Section 2), which leads us to draft specific strategies for different regions of the BD parameter space (Section 3). We also find it useful (while keeping in mind the uncertainties on such estimates) to derive the contribution of the population of $\mathrm{BD}$ primaries to the expected number of transiting habitable planets sufficiently close to be characterized through occultation spectroscopy, regardless of the type of their primary (Section 4). We examine the performance of occultation spectroscopic characterization for a habitable planet eclipsing a BD over the whole BD parameter space (Section 5). We then review the literature on the formation of terrestrial planets in the $\mathrm{HZ}$ of BDs and discuss BD HZs. Finally, in Section 7 we outline the key points relevant to the fast-track roadmap of time-resolving characterization of habitable exoplanets.

\section{OBSERVATIONAL CHARACTERISTICS OF HABITABLE PLANETS ECLIPSING A BROWN DWARF}

The detectability of eclipsing habitable planets around BDs has already been considered and attempted (Caballero \& Rebolo 2002; Caballero 2010): because of the small radius of the BD, putative terrestrial planets cause $1 \%-5 \%$ transit depths. Blake et al. (2008, hereafter BL08) additionally note that the small orbital radius of a habitable planet around a BD (a few times $10^{-3} \mathrm{AU}$ ) increases its likelihood of transiting. They also address

\footnotetext{
11 So, put another way, we simply attempt to push the astrobiological "follow
} the (liquid) water" philosophy to one of its many limits.

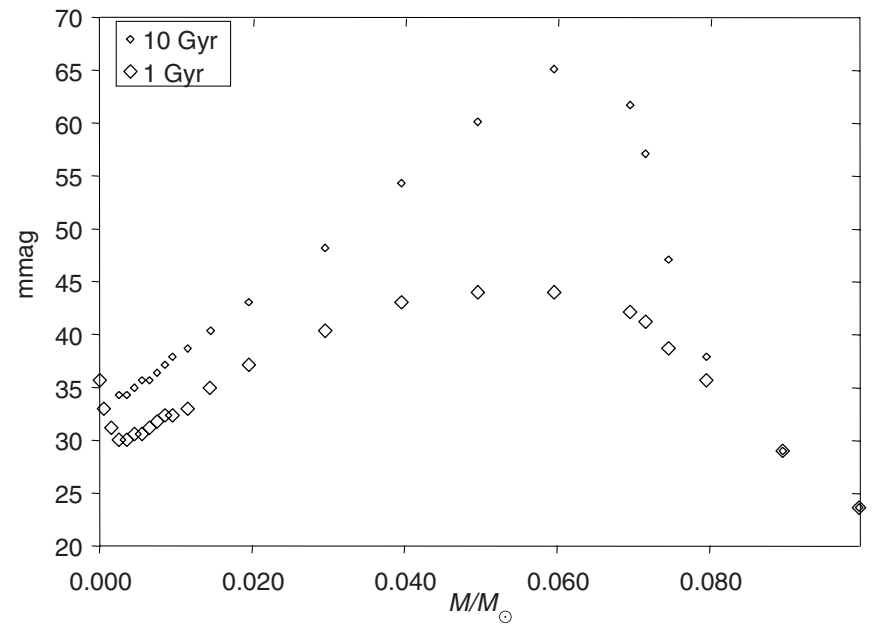

Figure 2. Transit depth of a $1.8 R_{\oplus}$ planet as a function of the brown dwarf's (BD) mass for different ages of the BD.

issues such as the convenient ruling-out of background blend false positives thanks to the high proper motion of a nearby BD population.

Figure 1 shows the orbital periods (in hours) of the effective HZs. We must caution here that the formula used for computing the HZ (Selsis et al. 2007b) is in principle valid for photospheric temperatures down to $3700 \mathrm{~K}$ (fit to models of the Earth around F-G-K dwarfs). For the lower photospheric temperatures of M dwarfs in Belu et al. (2011) the correction for photospheric temperature was fixed at the $3700 \mathrm{~K}$ value, and we do the same here: the scaling of the HZ is performed only through the luminosity of the primary (discussion of BD HZ in Section 6).

Ford \& Rasio (2006) show that if the planets undergo circularization then the limit of the possible orbital distances is twice the Roche limit, but if the planets undergo migration (such as in a disk) with a circular orbit, they can have orbital distances up to the Roche limit. We therefore also overplot in Figure 1 the orbital period at the Roche limit $P_{\text {Roche }}$ using the gravitation-only formula for the Roche $a_{\text {Roche }}$ limit used in BL08 (citing Faber et al. 2005 and Paczyński 1971). Since $a_{\text {Roche }} \propto$ $M_{\mathrm{BD}}^{1 / 3}$ (the BD's mass),

$$
P_{\text {Roche }}=\sqrt{4 \pi \frac{a_{\text {Roche }}^{3}}{G\left(M_{\mathrm{BD}}+M_{\mathrm{P}}\right)}}
$$

is fairly constant with the BD's mass because the terrestrial planet's mass $M_{\mathrm{P}} \ll M_{\mathrm{BD}}$. $G$ is the gravitational constant. For all tidal migration aspects (here and hereafter), see Section 4.2.

While photometrically monitoring SIMP J013656.5+093347 for intrinsic variability, Artigau et al. (2009) detect a $50 \mathrm{mmag}$ deep transit-like event in the $J$ band, with a precision of $5 \mathrm{mmag}$ over 5 minute bins (1.6 m telescope at Observatoire du Mont Mégantique). The simultaneous monitoring in another band yielded a different depth of the event, which, they conclude, would not be the case if an opaque body such as a planet were masking the BD (the signature of a transit is gray). Therefore, transit detection around a BD ideally involves simultaneously monitoring in two different bands.

For reference, Figure 2 shows the depth in mmag of the transit of a $1.8 R_{\oplus}$ planet as a function of the BD mass for different ages of the BD, with BD radii values from Baraffe et al. (2003, COND03 model). 




Figure 3. Minimal habitable planet eclipse duration (in minutes), as a function of the brown dwarf's (BDs) mass, for different ages of the BD, without and with tidal migration (optimistic planet formation at $10 \mathrm{Myr}$ considered). For masses below the plotted low mass bounds the radiative habitable zone is entirely below the Roche limit or below the minimal asymptotic final tidal-migration orbit (no effective planetary habitable zone).

BDs are also fast rotators. As they contract and cool down on sub-gigayear timescales their rotation speeds increase. We therefore note here that if the BD is significantly oblate and the planet's orbit is aligned with the BD's spin, this could reduce the transit depth because of equatorial gravity darkening (mention of this phenomenon in Herbst et al. 2007). For BDs older than 1 Gyr and heavier than $0.04 M_{\odot}$ Bolmont et al. (2011, Figure 1) predict rotation periods below $1 \mathrm{hr}$ (for reference, the rotation period of Jupiter is $\sim 10 \mathrm{hr}$ and its flattening 0.06). However, the authors recognize that measured BD rotational velocities available to fit their model are scarce, so caution is appropriate for this matter ${ }^{12}$.

In the case of unresolvable binary BDs (such as 2MASS 0939-2448 AB; Leggett et al. 2009), an Earth-like planet around the brightest component (S-type orbit) still produces a $3.5 \%$ (40 mmag) deep transit in the combined photometry. For other unresolvable binary BDs, if both feature an effective HZ, one survey actually can monitor two HZs simultaneously. ${ }^{13}$

The required cadence of observation is set by the expected minimal duration of a habitable planet eclipse around a BD (Figure 3):

$$
\tau_{\min }=P_{\mathrm{HZ} \min } \frac{1}{\pi} a \sin \frac{\sqrt{1-b^{2}} R_{\mathrm{BD}}}{\max \left(a_{\mathrm{HZ} \text { in }} ; a_{\mathrm{Roche}} ; a_{\mathrm{tides}}\right)},
$$

where $P_{\mathrm{HZ} \text { min }}=\max \left(P_{\mathrm{HZ} \text { in }} ; P_{\text {Roche }} ; P_{\text {tides }}\right)$ is the effective minimal habitable circular orbital period. $P_{\mathrm{HZ}}$ in is the orbital period at the inner limit $a \mathrm{HZ}$ in of the radiative habitable zone (HZ in), which is established for a planetary body. $P_{\text {Roche }}, P_{\text {tides }}$ and $a_{\text {Roche }}, a_{\text {tides }}$ are defined in the same way, at the Roche limit and for tidal migration, respectively. $R_{\mathrm{BD}}$ is the BD's radius, and

\footnotetext{
12 Also see Leconte et al. (2011). Beyond this article's title, this reference provides separate modeling of rotational deformation free from an exterior gravitational influence for BD mass range objects (J. Leconte 2013, private communication).

13 Also see Eggl et al. (2012). An Analytic Method to Determine Habitable Zones for S-Type Planetary Orbits in Binary Star Systems.
}

$b$ is the median impact factor where

$$
b=\frac{\max \left(a_{\mathrm{Roche}} ; a_{H Z \text { in }}\right)}{R_{\mathrm{BD}}+1.8 R_{\oplus}} \cos \left[\frac{\pi}{4}+\frac{1}{2} a \cos \frac{R_{\mathrm{BD}}+1.8 R_{\oplus}}{\max \left(a_{\mathrm{Roche}} ; a_{H Z \mathrm{in}}\right)}\right]
$$

for a $10 M_{\oplus}, 1.8 R_{\oplus}$ planet, although this median does not take into account the decrease in eclipse depth for high impact factors. ${ }^{14}$ If the BD is oblate and the planet's orbit is aligned with the BD's spin, the duration of the eclipse will be larger.

Finally, the fast rotation of BDs separates the transit signal cadence from rotation-induced variability. BDs are thought to feature evolving inhomogeneities in their cloud deck (weather), likely to generate variability in a manner similar to that of star spots (Knapp et al. 2004). The corotation distance is the orbit at which a planet's angular speed matches the BD's rotation. Thus as the rotation speed of the BD increases, the corotation distances moves inward with time. It becomes smaller than the Roche limit after 10-100 Myr, which is also the likely formation timescale of terrestrial planets around a BD (Bolmont et al. 2011). Thus, rotation-induced photometric variability of the BD primary would be in a totally different frequency regime than possible transit cadences. Still, if the evolution timescales of the inhomogeneities are of the order of the transit cadence (planet orbital periods), rotation-induced variability due to these features is very likely to increase the false alert rate (compare Figure 3 with Roche orbital periods).

\section{DETECTION STRATEGIES}

\subsection{The Importance of Completeness Assessment for Volume-limited Surveys}

For any given primary, a photometric monitoring of a given sensitivity can yield a single self-significant transit event candidate $^{15}$ down to a planet radius $R_{\mathrm{p}}$ (we consider a mean transit duration within our region of interest: the HZ). Monitoring this primary for a continuous duration $T$ implies that all potentially transiting planets with periods up to $T$, whatever their epoch (i.e., orbital phase), have been screened for. This can be dubbed " $100 \%$ completeness up to $T$ (and down to $R_{\mathrm{p}}$ )." Publishing the completeness of a volume-limited survey is particularly important when the final yield of detections is expected to be low, or even below unity. Indeed, the yield is necessary for planning follow-up eclipse spectroscopic characterization (including building of dedicated facilities).

We now examine specific strategies for different regions of the BD parameter space.

\subsection{Ground-based, Single-night, Single-longitude, $100 \%$ Completeness}

If we ignore tidal migration (Section 4.2), the Roche-limited HZ (gray contour in Figure 1) extends to orbital periods shorter than the typical photometric night durations (e.g., but not limited to, airmass <2). The Roche limit is at $3.6 \mathrm{hr}$ for the $10 \mathrm{M}_{\oplus}$, $1.8 R_{\oplus}$ super-Earth considered in Figure 1 , at $4.5 \mathrm{hr}$ for an analog of the Earth, and at $5 \mathrm{hr}$ for a $0.1 M_{\oplus}, 0.5 R_{\oplus}$ planet. This means that some BDs can be screened with $100 \%$ completeness

\footnotetext{
14 Also note that Equation (1) assumes the transit to start when the center of the planet touches the limb of the primary, whereas Equation (2) assumes transit start at first contact. Assessing the error induced by all these imprecisions in modeling is beyond the scope of the present work. 15 By default and unless mentioned otherwise no shift adding of multiple observation nights (to increase the signal-to-noise ratio, $\mathrm{S} / \mathrm{N}$ ) is considered in this paper (more on this in Section 3.3).
} 
Table 1

Photon Noise-only Signal-to-noise Ratio $(\mathrm{S} / \mathrm{N})$ of the Detection of a Single-transit Event in $J$ Band for a Non-exhaustive List of BDs with $P_{\mathrm{HZ}}$ out $\leqslant 8$ hr

\begin{tabular}{|c|c|c|c|c|c|c|c|c|c|}
\hline & \multicolumn{2}{|c|}{$P_{\mathrm{HZ}}(\mathrm{hr})$} & \multirow{2}{*}{$\begin{array}{c}\tau_{\min } \\
\text { (minutes) }\end{array}$} & \multirow{2}{*}{$\begin{array}{l}\text { Depth } \\
(\%)\end{array}$} & \multirow{2}{*}{$\begin{array}{l}\mathrm{mag} \\
(J)\end{array}$} & \multirow{2}{*}{$\begin{array}{l}\text { Photons } \mathrm{s}^{-1} \mathrm{~m}^{-2} \mathrm{a} \\
\qquad\left(\times 10^{3}\right)\end{array}$} & \multirow{2}{*}{$\begin{array}{c}\text { Photons, per } 1 / 2 \text { Transit } \\
\text { at } \mathrm{HZ} \text { in }\left(\times 10^{3}\right)\end{array}$} & \multirow[t]{2}{*}{ S/N per Transit } & \multirow{2}{*}{$\begin{array}{c}\text { Geom. Prob. HZ out } \\
(\%)\end{array}$} \\
\hline & in (Roche) & out & & & & & & & \\
\hline SDSS J1416+13 B & 3.42 & 5.99 & 12.2 & 3.8 & $17.35^{\mathrm{b}}$ & 3.8 & 600 & 21 & 16 \\
\hline 2MASS 0939-2448 A & 3.42 & 6.52 & 12.5 & 4.0 & $16.1^{\mathrm{c}}$ & 11 & 1800 & 34 & 14 \\
\hline $\mathrm{AB}$ & & & & 3.5 & 15.98 & 13.4 & 2200 & & \\
\hline CFBDS J005910-011401 & 3.42 & 5.45 & 16.1 & 3.6 & $18.06^{\mathrm{d}}$ & 1.98 & 416 & 17 & 20 \\
\hline
\end{tabular}

Notes. $P_{\text {HZ out }}$ is the orbital period at the outer edge of the radiative habitable zone. No tidal migration is considered here. $3.5 \mathrm{~m}$ class telescope. We want the cadence to be half the minimal possible duration of the transit $\tau \min$ (at least one complete exposure taken during the transit).

a 0.82 transmission of the filter included.

${ }^{\mathrm{b}}$ Burningham et al. (2010).

${ }^{\mathrm{c}}$ The magnitude of the A component alone was estimated using values from Table 2, and it was checked that when doing the same with the B component the fluxes in the next column add up.

${ }^{\mathrm{d}}$ Scholz et al. (2009). For references on the remaining parameters of the targets, see Table 2.



Figure 4. Planet orbital periods for which transit screening completeness is $100 \%$, for two adjacent full monitoring nights each of usable duration $n$ (in the case of just one monitoring night the maximum orbital period, for which completeness is $100 \%$, is of course only $n$-diamonds).

from the ground, in just a single observing night, with a single telescope at a single longitude-a remarkable efficiency.

When such a candidate is detected up to three subsequent follow-up nights are required: the first for determining a period and confirming the alert, and the second for confirming the period and the periodic nature of the signal. An additional shifted third night can help rule out submultiples of the initial period. A tradeoff in the shift has to be determined since the greater the shift the greater the build-up of ephemeris uncertainties.

We explore the increase in orbital period screening by monitoring for one adjacent night (Figure 4). For instance in the case of a $9 \mathrm{hr}$ useful night, the addition of one adjacent observation night yields $100 \%$ completeness for planet orbital periods up to $11 \mathrm{hr}$, and also for orbital periods between $\sim 15$ and $\sim 16.5 \mathrm{hr}(\sim 39 \%$ increase $)$.
Table 1 gives the expected, photon noise-only signal-to-noise ratio $(\mathrm{S} / \mathrm{N})$ of the detection of a single transit event with a $3.5 \mathrm{~m}$ class telescope in the $J$ band for several nearby BDs with $P_{\mathrm{HZ} \text { out }} \leqslant 8 \mathrm{hr}$. The integration time is derived from the minimal possible duration for the transit of a habitable planet (i.e., a transit at the inner edge of the habitable zone- $-\mathrm{HZ}$ in). We further halve this integration time to take into account alternating between two bands (see the Artigau et al. observation mentioned above), in case no dichroic is available. We consider the overhead per one-forth back filter switching to be $40 \mathrm{~s}$ (case for WIRCAM (Wide-field Infra-Red Camera) on the Canada-France-Hawaii Telescope-(CFHT)).

In the last column, we also give the geometric likelihood of transit at the outer limit of the HZ (i.e., the lower limit on the transit likelihood of habitable planets around these BDs; evidently, no primary types have higher habitable planet transit probability than the BD type.

To conclude, we note that BDs are currently being photometrically monitored with IR telescopes for increasingly extended continuous periods in the frame of atmospheric (weather) and evolution tracks research. We therefore call to this community to integrate the science case presented in this subsection in the evolution and further expansion of their field.

\subsection{Ground, Multiple Nights}

BD HZs extend up to 10 days of orbital period (Figure 2). BL08 suggested the use of a redundant, longitude-distributed network of telescopes for continuous photometric monitoring, such as the Las Cumbres Global Telescope (LCOGT) network. ${ }^{16}$ We have executed a test of such longitude distributed observation in early 2011. One $z=17$ target was scheduled for $13.5 \mathrm{hr}$ of continuous monitoring, involving the two $2 \mathrm{~m}$ telescopes of the LCOGT in Hawaii and Australia and the $2 \mathrm{~m}$ Himalaya Chandra Telescope (HCT) with the Himalaya Faint Object Spectrograph and Camera.

Meteorological conditions enabled only observations from $\mathrm{HCT}$, and the overall environmental conditions for that observation caused a high background level. Therefore, the $\mathrm{S} / \mathrm{N}$ of the final light curve (not shown) for this very faint target was too low for exploitation.

In conclusion, for the moment such a $2 \mathrm{~m}$ far red opticalclass network may not be yet sufficiently longitude-redundant for robustness against environmental variability. Also, slightly

\footnotetext{
16 www.lcogt.net
} 
brighter-on-average targets may relax the constraints on environmental conditions. However, these targets would have longer $\mathrm{HZ}$ outer limit periods, therefore requiring longer monitoring in order to achieve complete HZ screening. For instance already for the present test target the longest habitable period was longer than the 13.5 continuous hours we were able to secure. Longer monitoring means more different observatories are stringed together for such an observation. The red spectral energy distribution of BDs also advocates for extending the equipment of the 2 m class collectors worldwide with $J-H-K$ detectors. Last, taking into account the meteorological forecasts at the different observatories and triggering the observing sequence in a target-of-opportunity fashion could be investigated.

If the continuity is disrupted before the longest habitable period can be covered, a scheduling algorithm can enable us to optimize the completeness of the screening of a given target (e.g., Saunders et al. 2008). The completeness may reach near $100 \%$ but with a significant increase in observation time cost. However, such multiple observations enable us to search for shallower transits and/or primaries with increased variability using phase-folding search techniques and accounting for the subsequent introduction of correlated (red) noise (von Braun et al. 2009). Note that flare variability can be a real challenge for phase folding in M dwarfs' light curves.

Last, Blake \& Shaw (2011) have shown recently that, following the quality of the site, preciptable water vapor (PWV) variability can induce $5 \mathrm{mmag}$ variations in the $z$ band on an hour timescale; however, they indicate that PWV can be monitored through global positioning system signals.

\subsection{Intermediate Cases}

For the BDs with outer habitable periods between $\sim 8$ and $\sim 20 \mathrm{hr}$ (i.e., the cases intermediate to those addressed in Sections 3.2 and 3.3 above), one would require a network such as the one described above but operating in the infrared. Such coordinated observations between telescopes usually operated through time allocation committees may prove difficult to set up (considering the very high pressure on these telescopes and constraints on mutual telescope observing coordination). Therefore, in the frame of a cohesive grand strategy for ground detection of habitable planets eclipsing BDs, coordinated observations are likely only as a second step, after single-telescope, single-night observations on cooler targets are first demonstrated (Section 3.2 above). If no coordinated observation can be set up, observations have to be spread throughout the observing season of the target, arranging them so that together they satisfactorily cover the time-folded range of orbits that is sought, significantly increasing the total cost in telescope time. See also Berta et al. (2012) for a related study deriving from the MEARTH survey for habitable planets transiting $M$ dwarfs. This study includes analytical tools for integrating "lone transit events" (from different telescopes using different filters at different observatories) into coherent planet candidates.

The optimal approach for screening these intermediate cases is a dedicated monitoring program from space, where uninterrupted monitoring can be achieved. Since 2011 August the Spitzer Warm Mission Exploration Science Program 80179 "Weather on Other Worlds: A Survey of Cloud-Induced Variability in Brown Dwarfs" (PI: Metchev) has been monitoring BDs from a list of 44 targets $(873 \mathrm{hr} \text { awarded in total })^{17}$ one

\footnotetext{
17 http://sha.ipac.caltech.edu/applications/Spitzer/SHA//\#id= SearchByProgram $\&$ DoSearch $=$ true $\&$ SearchByProgram. field.program $=80179$
}

after another for a minimum of 21 continuous hours each Unfortunately none of the 25 targets observed up until now are at or beyond 7 pc. Also, observing simultaneously in both channels of Warm Spitzer (3.6 and $4.5 \mu \mathrm{m}$ ) is not possible because the arrays of each channel see different non-overlapping parts of the sky. Therefore, a prospective interlaced mode is not up for consideration at the cadence required by both intrinsic variability studies and exoplanet detection.

\section{EXPECTED NUMBER OF NEARBY ECLIPSING HABITABLE PLANETS}

\subsection{Previous Study}

Due to $\mathrm{S} / \mathrm{N}$ constraints, habitable planets can be searched for biosignatures with eclipse spectroscopy only out to a limited distance. Belu et al. (2011, Figure 8) find that at 6.5 pc emission biosignatures can be detected within JWST's baseline lifetime only around primaries of spectral types later than $\sim \mathrm{M} 5$. The $\mathrm{S} / \mathrm{N}$ scales as the planetary radius squared and inversely with the distance. Also, considering a specific spectral signature a given planet might exhibit (and which would require confirmation or rejection), the signature's $\mathrm{S} / \mathrm{N}$ scales linearly with the strength of the spectral feature (number of atmospheric scaleheights for primary eclipse and brightness temperature depth for the secondary), and with the inverse of the resolution.

Belu et al. further showed (Figure 19 therein) that in a $\sim 7 \mathrm{pc}$ volume, ${ }^{18}$ the total expected number of habitable eclipsing planets is $\sim 0.3$ for the M5-M9 dwarf primaries population (considering the recent Bonfils et al. 2013 lower bound for $\eta_{\oplus}$ : 0.41). Note that Selsis et al. (2007b) pointed out that a primary may in principle host several habitable planets (as was perhaps the case for the Sun 4 Gyr ago, when Venus, the Earth, and Mars were potentially habitable). Therefore, the case of a final $\eta_{\oplus}>1$ is not to be discarded. For the compact habitable zones of BDs $\left(10^{-3} \mathrm{AU}\right.$ scale), further studies are needed to address the stability of multiple planets in this zone, and their possibility to form and/or migrate (see the next subsection).

\subsection{Present Study}

We now extend the previous study to T, L, and Y dwarfs; some young BDs could exhibit $M$ spectral type (and vice versa): they would have been included in the previous study. Table 2 lists the known BDs likely within 7 pc. This table was compiled from RECONS (www.recons.org), SIMBAD, and other recent discoveries. For each object we have collected from the literature estimates of photospheric temperature, mass, radius, and luminosity. The missing values were interpolated from the COND03 evolutionary grids (Baraffe et al. 2003) using the available parameters.

Table 2 contains two binary systems. GJ 845 Bab ( $\varepsilon$ Indi $\mathrm{Bab})$ is a binary BD system, with a separation between the components of at least 2.1 AU (Volk et al. 2003). The outer limit of the habitable zone for each of the two components (S-type orbits) is 0.01 and $0.005 \mathrm{AU}$, respectively, so orbital stability at these distances is not an issue. On the other hand, 2MASS 0939-2448 is likely a dissymmetric close binary (Burgasser et al. 2008; Leggett et al. 2009) with a separation of under 0.03 AU. Holman \& Wiegert (1999) have derived semi-major axis upper limits for dynamically stable orbits

\footnotetext{
18 Volume limit of the 100 nearest objects at that time, RECONS
} (www.recons.org). 
Table 2

Total Expected Number of Eclipsing Habitable Planets around Nearby L, T, Y Dwarfs

\begin{tabular}{|c|c|c|c|c|c|c|c|c|c|c|c|}
\hline & \multirow{2}{*}{$\begin{array}{c}\text { Distance } \\
\text { (pc) }\end{array}$} & \multirow{2}{*}{$\begin{array}{c}T \\
(\mathrm{~K})\end{array}$} & \multirow[t]{2}{*}{$M / M_{\odot}$} & \multirow[t]{2}{*}{$R / R_{\odot}$} & \multirow{2}{*}{$\begin{array}{c}L / L_{\odot} \\
(\log )\end{array}$} & \multirow[t]{2}{*}{ Reference } & \multirow[t]{2}{*}{$\mathrm{HZ}_{\text {Roche }}$} & \multicolumn{2}{|c|}{ HZ $\mathrm{Z}_{\text {asymp. }}$} & \multicolumn{2}{|c|}{ Trans. Prob. } \\
\hline & & & & & & & & $1 \mathrm{Myr}$ & $10 \mathrm{Myr}$ & $1 \mathrm{Myr}$ & $10 \mathrm{Myr}$ \\
\hline WISE $1541-2250$ & $2.8^{\mathrm{a}}$ & 350 & 0.011 & 0.01 & -6.88 & Cushing et al. 2011 & 0 & & & & \\
\hline $\begin{array}{r}\text { GJ } 845 \mathrm{~B} \mathrm{a} \\
\mathrm{b}\end{array}$ & 3.6 & $\begin{array}{r}1320 \\
910\end{array}$ & $\begin{array}{l}0.065 \\
0.050\end{array}$ & $\begin{array}{l}0.0805 \\
0.0825\end{array}$ & $\begin{array}{l}-4.699 \\
-5.232\end{array}$ & King et al. 2010 & $\begin{array}{r}1 \\
0.95\end{array}$ & & 0.64 & & 0.030 \\
\hline SCR $1845-6357$ B & 3.85 & 950 & 0.039 & 0.091 & -5.1 & Kasper et al. 2007 & 1 & & 0.27 & & 0.019 \\
\hline UGPS $0722-05$ & 4.1 & 505 & 0.005 & 0.10 & -6.13 & Leggett et al. 2012 & 0.79 & & & & \\
\hline DEN 0817-6155 & 4.9 & 950 & 0.04 & 0.089 & -3.53 & Artigau et al. 2010 & 0.98 & & & & \\
\hline DEN 0255-4700 & 5.0 & 1300 & 0.035 & 0.09 & -4.62 & Stephens et al. 2009 & 1 & 0.55 & 0.74 & 0.023 & 0.033 \\
\hline $\begin{array}{r}\text { 2MASS 0939-2448 A } \\
\text { B }\end{array}$ & 5.3 & $\begin{array}{l}700 \\
500\end{array}$ & $\begin{array}{l}0.038 \\
0.024\end{array}$ & $\begin{array}{r}0.085 \\
0.09\end{array}$ & $\begin{array}{l}-5.8 \\
-6.3\end{array}$ & Leggett et al. 2009 & $\begin{array}{r}0.52 \\
0\end{array}$ & & & & \\
\hline WISE $1741+2553$ & $5.5^{\mathrm{a}}$ & & & & & d & 0.79 & & & & \\
\hline 2MASS 0415-0935 & 5.7 & 947 & 0.01 & 0.12 & -5.0 & Del Burgo et al. 2009 & 1 & 0.24 & 0.14 & 0.021 & 0.011 \\
\hline GJ $229 \mathrm{~B}$ & 5.8 & 950 & 0.038 & 0.094 & -5.2 & Geißler et al. 2008 & 1 & & 0.08 & & 0.006 \\
\hline GJ $570 \mathrm{D}$ & 5.9 & 948 & 0.019 & 0.11 & -5.0 & Del Burgo et al. 2009 & 1 & 0.55 & 0.14 & 0.051 & 0.010 \\
\hline SIMP J013656.5+093347.3 & $6^{\mathrm{b}}$ & 1200 & 0.044 & 0.097 & -5.25 & Artigau et al. 2009 & 0.96 & & & & \\
\hline 2MASS $0937+2931$ & 6.1 & 950 & 0.054 & 0.08 & -5.33 & Leggett et al. 2010 & 0.87 & & & & \\
\hline WISE $0254+0223$ & $6.1^{\mathrm{a}}$ & 660 & 0.01 & 0.11 & -5.7 & Kirkpatrick et al. 2011 & 0.95 & & & & \\
\hline \multirow[t]{6}{*}{ WISE $1738+2732$} & $7^{\mathrm{c}}$ & 350 & 0.019 & 0.093 & -6.94 & Cushing et al. 2011 & 0 & & & & \\
\hline & & & & & & & & & & 0.09 & 0.11 \\
\hline & & & & & & & & \multicolumn{4}{|c|}{ Expected \# } \\
\hline & & & & & & $\times 0.41_{-0.13}^{+0.54}=$ & $0.78_{-0.25}^{+1}$ & & & $0.036_{-0.012}^{+0.048}$ & $0.078_{-0.014}^{+0.06}$ \\
\hline & & & & & & & & \multicolumn{4}{|c|}{$\%$ probability of at least 1 occurrence } \\
\hline & & & & & & & $56_{-13}^{+31}$ & & & $3.9_{-1.2}^{+4.9}$ & $4.5_{-1.4}^{+5.6}$ \\
\hline
\end{tabular}

Notes. $\mathrm{HZ}_{\text {Roche }}$ is the fraction of circular orbits that are in the habitable zone but outside a $10 M_{\oplus}$ Roche limit (uniform distribution in radius). Similarly $\mathrm{HZ}$ asymp is the fraction, from the remaining habitable zone, where planets can exist at the end of the tidal migration process for two different planet formation ages. The last two columns give the corresponding transit probability of the median orbit

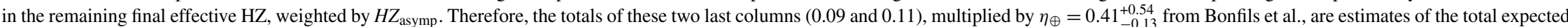
number of habitable planets transiting BDs in this volume (see text for detailed justification).

Distances (pc) are RECONS parallaxes unless mentioned otherwise. When parallaxes were not available, we used photometric distances. Values in italic are from the reference. The remaining non-italic parameters (among photospheric temperature $T$, mass $M$, radius $R$ and luminosity $L$ ) are interpolated from COND03 grids using the parameters from the reference. Note that the purpose of this table is to compute some ensemble averages; therefore the values of the BD parameters should not be reused for the study of individual objects, since the uncertainties on most parameters are quite large (e.g. spectroscopic/photometric distance estimates), and because of ongoing refined observations (e.g. parallaxes)

a Kirkpatrick et al. (2011), parallax.

${ }^{\mathrm{b}}$ Faherty et al. (2009).

${ }^{c}$ Average of quite dissimilar photometric distance (Kirkpatrick et al. 2011) and spectroscopic distance (Cushing et al. 2011).

${ }^{\mathrm{d}}$ For this BD, interpolation of the grids to the 2MASS $J$ and $H$ magnitudes (Kirkpatrick et al. 2011; Scholz et al. 2011) did not converge (as it was more the case for WISE J0254+0223). We therefore use here the values of the only other T9 BD in the sample, UGPS 0722-05. 
around each component of a binary. The outer habitable orbits around each likely component of 2MASS 0939-2448 (0.0029 and $0.0016 \mathrm{AU}$, respectively, or 7.2 and 3.8 times the radii of their respective primaries) are indeed dynamically stable.

We then calculate for each BD the fraction of circular orbits that are in the habitable zone but outside the Roche limit (column $\mathrm{HZ}_{\text {Roche }}, 10 M_{\oplus}$ planet).

When computing geometric transit probabilities it is usually assumed that the probability of a planet occupying a given orbit is flat across the available parameter space, e.g., the $\mathrm{HZ}$. However, given the close proximity of BDs' HZs, tidal interactions between the planet and BD act to modify a planet's orbit after its formation, with consequences for its transit probability.

Bolmont et al. (2011) studied the tidal evolution of planets orbiting BDs (previously referred to here as tidal migration). The basic concept is as follows. We already mentioned the gradual reduction of the corotation distance (Section 2). This is important because a planet's position with respect to corotation determines the direction of tidal migration. Given that BDs' corotation distances shrink in time, almost all planets that survive around BDs experience outward tidal migration. Thus, there is a parameter-dependent orbital radius inside of which planets should not exist. The asymptotic limit is reached after 10-100 Myr. The ages of observed BDs are known with precisions equal to or larger than $0.1 \mathrm{Gyr}$, and all of the BDs in Table 3 have age estimates larger than or equal to $100 \mathrm{Myr}$. We therefore proceed and apply the asymptotic limit model to all the BDs in Table 2.

The key parameters that determine this limit are the BD and planet masses and their internal dissipation rates (Bolmont et al. 2011). From this reference we use Figures 6 and 8 which give the asymptotic limit (semi-major axis) for planets around BDs of different masses (for a $1 M_{\oplus}$ planet forming at 1 and $10 \mathrm{Myr}$, respectively). For the remaining BDs in Table 2 that have $\mathrm{HZ}_{\text {Roche }}>0$ ( $3 \mathrm{BDs}$ do not) we give the non-null fractions of circular orbits that are in the habitable zone but with semi-major axis larger than the asymptote (column $\mathrm{HZ}_{\text {asymp. }}$ ).

Finally, for the remaining BDs that still feature the above effective final habitable zone, we compute the transit likelihood at the middle of the zone (with the same working assumption $\eta_{\oplus}=1$ ) and we weight (multiply) this likelihood by $\mathrm{HZ}_{\text {asymp. }}$ thus obtaining a final transit probability (column Transit. Prob.). The justification for this weighting is the following. These individual likelihoods are then multiplied by the recently directly measured $\eta_{\oplus}$ within the $\mathrm{M}$ dwarf primary population, $0.41_{-0.13}^{+0.54}$ (Bonfils et al. 2013, lower limit, as per sensitivity of the technique to the whole range of habitable planet masses). Weighting our individual transit likelihoods by $\mathrm{HZ}_{\text {asymp. is a }}$ valid approach because the habitability of individual planets in the sample of Bonfils et al. is based on radiative (from the primary) considerations alone, and their primary population is not subject to the limitations included in the $\mathrm{HZ}_{\text {asymp. factor }}$ (Roche limit and tidal migration). Their $\eta_{\oplus}$ contains only information on planet orbital density as a function of the primary; it can validate formation and migration models, the latter excluding tidal migration because the habitable distances around $\mathbf{M}$ dwarfs are too large for this mechanism. It is this formation- and migration-other-than-tidal-planet density function of Bonfils et al. that we extrapolate to BDs. We must caution, however, that the figures in Bolmont et al. (2011) seem to indicate that the tidal migration mechanism tends to redistribute orbits (i.e., change the density of orbits, either shepherd- ing them together or dispersing them, depending on the initial conditions).

The final expected number of eclipsing habitable planets around BDs within $7 \mathrm{pc}$ is given at the bottom of Table 2 . A more significant number is the probability for a survey to yield at least one transiting habitable planet in this volume (also in Table 2). Assuming the optimistic scenario of late planet formation around BDs (i.e., at $10 \mathrm{Myr}$ ), the survey of the corresponding 6 BDs in Table 2 has a $4.5_{-1.4}^{+5.6} \%$ chance of yielding at least one habitable eclipsing planet.

Given the recent nature of the work on tidal migration, we also include the significantly more optimistic figures when tidal migration is not considered. The probability for the survey of the above 14 BDs with Roche-limited-only HZs to yield at least one transiting habitable planet likely within $7 \mathrm{pc}$ is then $56_{-13}^{+31} \%$.

\subsection{Discussion}

Thus, to include BD primaries in the search for nearby, eclipse-characterizable habitable planets is to increase the expected number of occurrences $\sim 2.5$-fold (when compared with the late-M-dwarf-only search).

The results on tidal migration depend on some parameters that are unknown/poorly constrained for our nearby BDs, such as the dissipation factor in the $\mathrm{BD}$ or the initial rotation rate. There are also various rotation braking mechanisms that are not considered. This is to be combined with the uncertainties on the parameters of which we give the estimates (mass, age). Will future refinements of tidal migration modeling enable us to gain back the order of magnitude between the Roche-only limitation of the habitable zone and the one by tidal migrations? Or will it completely rule out habitable planets around BDs? What about other mechanisms for late migration or the frequency of late planet scattering?

The planet's mass is also a strong factor for tidal migration, with planets of $0.1 M_{\oplus}\left(0.5 R_{\oplus}\right)$ hardly experiencing any effect. Unfortunately, the performance of the subsequent search for spectral signatures scales with the square of the planet's radius. But this should also remind us that the volume limit we used for our list is an average estimation of spectroscopic characterization capability with the JWST; particularly favorable cases (strong spectral signatures, planets close to the inner limit of the habitable zone, etc.) may be characterizable further away. Planet detection surveys should therefore plan a significant margin on these volume estimates (and the number of targets scales with the cube of the distance).

Also note that only lower bounds on the local space density of BDs are presently available (Kirkpatrick et al. 2011). The final BD detection count from the ongoing processing of the WISE data is expected to be $\sim 1000$ BDs, which should double or triple $^{19}$ the number of known primaries within 25 light years (7.6 pc). Therefore, our values should be considered lower limits.

\section{FUTURE CHARACTERIZATION}

We now consider habitable planet secondary eclipse spectroscopy performance around a BD. We reprise our previous such study around F-M dwarves with the JWST (see Belu et al. 2011 for a detailed description of the modeling). BDs may not exhibit significant near-UV flux. Therefore, a (biotic) $\mathrm{O}_{2}$ atmosphere on a BD exoplanet may not generate $\mathrm{O}_{3}$ (ozone) in its

\footnotetext{
19 As of 2009 December; NASA WISE Launch Press Kit.
} 

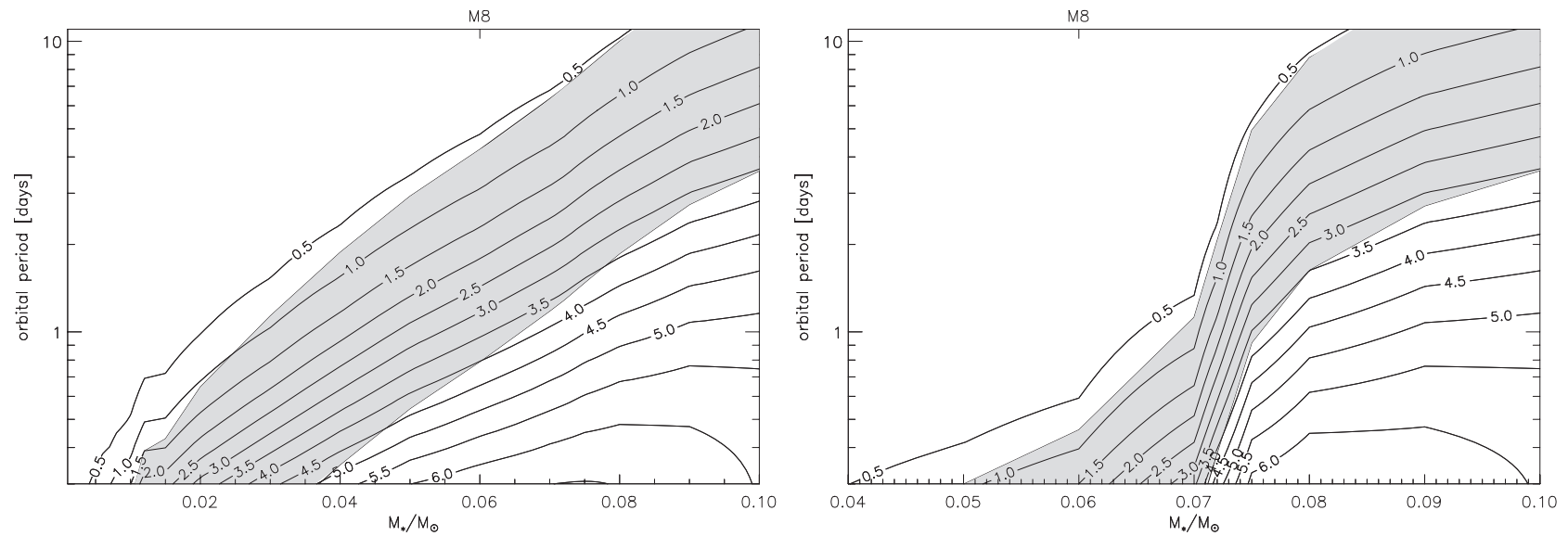

Figure 5. Signal-to-noise ratio $(\mathrm{S} / \mathrm{N})$ on the detection of a spectral feature in emission (secondary eclipse spectroscopy), at $10 \mu \mathrm{m}$, as a function of the mass of the brown dwarf and the orbital period of the planet. The spectral feature has a brightness temperature depth of $30 \mathrm{~K}$ and is $0.1 \mu \mathrm{m}$ wide (i.e., $R=100$ ). The planet is a 1.8 Earth-radii super-Earth, and the system is situated at $6.7 \mathrm{pc}$. The observations of 90 eclipses are summed for this result. The age of the brown dwarf is $1 \mathrm{Gyr}$ (left) and $10 \mathrm{Gyr}$ (right, note the different abscissa scale). The grayed area is the habitable zone (Section 2). The S/N scales linearly with the square root of the number of summed eclipses (if no correlated noise), with the square of the planet's radius, with the brightness temperature depth, with the inverse of the distance in parsecs, and with the inverse of the resolution.

stratosphere, which is a convenient $\mathrm{O}_{2}$ detection proxy around $10 \mu \mathrm{m}$ (location of thermal emission from a body at habitable temperatures). The question of BD HZs and biosignatures is discussed at length in Sections 6.2 and 6.3. We therefore consider a fiducial spectral feature in emission (at $10 \mu \mathrm{m}$, brightness temperature depth of $30 \mathrm{~K}$, and $0.1 \mu \mathrm{m}$ wide, i.e., resolution $R=100)$.

The instrument considered is the Mid Infra-Red Instrument in the Low Resolution Spectroscopy (LRS) mode. For 1 and $10 \mathrm{Gyr}$ old BDs, Figure 5 shows the $\mathrm{S} / \mathrm{N}$ on the detection of our fiducial spectral feature from a 1.8 Earth-radii planet at $6.7 \mathrm{pc}$, summing the observations of 90 secondary eclipses. Program time cost per eclipse is twice the eclipse duration (at least $\sim 30$ minute, Figure 2), plus the 65 minute generic JWST slew time budget, every 10-70 hr (period of the planet). Note that even for the longest period planets ( $\sim 10$ days), 90 transits are well within the telescope's lifetime. We repeat here our comment from Belu et al. (2011): such a hypothetical observation, which would happen on only one (see Section 4) most interesting transiting system, represents a total telescope time only a magnitude larger than the longest exposures made until now with the Hubble Space Telescope (HST; Beckwith et al. 2006). Also note that the 1.8 Earth radii is an upper limit for habitability, but could be extremely optimistic in terms of initial mass available in a BD's protoplanetary disk for planet formation.

Despite the lower luminosity of the primary, hence the reduced photon noise, shorter orbital periods also mean shorter occultation durations (10-40 minutes). This curbs the gain one could have expected relative to the case around $M$ dwarfs. The discussion at the end of Section 2 on the rotation-induced variability of primary and transit detection also applies to this eclipse characterization follow-up.

One can see that atmospheric absorption features such as those presented here can be detected on habitable planets eclipsing BDs after a follow-up of a couple of months, for a cost of $<2.5 \mathrm{hr}$ of observation every 1-2 days, so on average about $1 \%$ of the five-year mission time of the JWST. This cost in mission time is about a factor of two better on average than for M dwarf habitable planets (Belu et al. 2011), and more importantly, spread over only one-fifth to one-tenth of the five-year mission time (whereas in the $\mathrm{M}$ dwarf case the required number of observations spreads over the entire mission lifetime, exposing them to the risk of dedicating time and acquiring data that ends up having insufficient $\mathrm{S} / \mathrm{N}$ if the JWST were to become inoperable too soon).

\section{DISCUSSION}

\subsection{Terrestrial Planet Formation and Orbital Evolution around $B D$ s}

BL08 and Bolmont et al. (2011) reviewed the literature on the likelihood of formation of habitable planets around BDs. There is ample evidence in favor of terrestrial planet formation around BDs: the same fraction of young BDs has circumstellar disks as do T Tauri stars (Jayawardhana et al. 2003; Luhman et al. 2005), and there is observed evidence of grain growth in BD disks (Apai et al. 2005). Of course, the exact outcome of the accretion process depends on the disk mass and mass distribution (Raymond et al. 2007; Payne \& Lodato 2007), which probably scales roughly linearly with the primary mass (Andrews et al. 2010). Regarding formation, see also Charnoz et al. (2010) for late accretion at the Roche edge of a debris disk.

If tidal migration influences are confirmed there should be no planets with orbital periods under $8 \mathrm{hr}$ orbiting them (Figure 3, black contours). Only extremely unlikely scenarios could permit such planets, such as unusually low dissipation factors; unusually high initial rotation rates; or very recent capture, migration, or formation. For instance, Figure 19 in Bolmont et al. (topmost panel for the lowest BD dissipation factor) shows a tremendous sensitivity to the initial semi-major axis when computing the final asymptotic one. In theory there could be an extremely narrow interval of initial positions for which the final asymptotic orbit is as close as desired to the Roche limit. This interval is likely $\ll 10^{-3}$ AU (the span of initial semi-major axes for which planets that migrate in are saved, whatever their final asymptotic semi-major axis).

However, we recall that the detection of hot Jupiters was proposed as feasible almost half a century before their actual detection (Struve 1952). To summarize, there are significant theoretical uncertainties associated with each of these questions and no certain answers at the present time. We are of the opinion that, given their exceptional detection advantages (one night, one location, $100 \%$ completeness) we should invest in the presented strategy. Additionally, whole-night monitoring 
of BDs may enable improved BD atmospheric studies. Therefore, planets transiting BDs with periods under $8 \mathrm{hr}$ have a high payoff/screening cost ratio.

\subsection{Habitability and $B D s$}

A radiative habitable zone ( $\mathrm{HZ})$, within which terrestrial planets can sustain surface liquid water, can be defined around BDs. The inner-edge of the $\mathrm{HZ}$ corresponds to an $\mathrm{H}_{2} \mathrm{O}$-rich atmosphere and the outer edge to a greenhouse efficient gas-rich atmosphere-most likely $\mathrm{CO}_{2}$. The inner limit is reached when the mean stellar flux absorbed by the planet is $300 \mathrm{~W} \mathrm{~m}^{-2}$ (runaway greenhouse threshold). Determining the location of the outer edge, which depends on the efficiency of $\mathrm{CO}_{2}$ as a greenhouse gas, will require specific climate modeling (1D and 3D), due to the strong overlap between the thermal emission of the BD and the molecular lines in the planet's atmosphere (e.g., Wordsworth et al. 2011). The full absorption of the continuum of $\mathrm{H}_{2} \mathrm{O}$ and $\mathrm{CO}_{2}$, and the absence of Rayleigh scattering will likely lead to a planetary albedo close to null (we take 0.1 in Section 5). Strong stratospheric warming and inefficient greenhouse is expected, possibly leaving the surface at a lower temperature than the globally perceived brightness temperature of the stratosphere. We are currently developing 1D and 3D codes suitable for BD planets. The contribution of internal heating due to tidal effects (Jackson et al. 2008 for stellar masses down to $0.1 M_{\odot}$ ) may also be significant.

Several threats against surface habitability exist within the $\mathrm{HZ}$ of BDs. One is the tidal-spin-orbit synchronization. Planets on circular orbits inside the $\mathrm{HZ}$ of $\mathrm{M}$ stars and BDs are expected to have a permanently dark hemisphere and a zero obliquity. If zonal and meridional heat transport is insufficient the water and the atmosphere can end as condensed caps at the poles and night side of the planet. Simulations for GJ $581 \mathrm{~d}$ (Wordsworth et al. 2011) show, however, that a dense atmosphere can provide enough heat transport to homogenize the temperature over the whole surface (see also Joshi 2003).

Another threat is the rapid cooling of the BD (Figure 5), which has two implications. The first one is that, coupled with the tidal migration outward drift, a planet remains habitable during only a fraction of the BD's life (more than 1 Gyr only for BDs > $0.04 M_{\odot}$; Bolmont et al. 2011). Nonetheless, life on Earth is thought to have existed within $1 \mathrm{Gyr}$ of its formation. Thus, although planets have a short habitable window around BDs, it is of great scientific interest to search for them (see Lopez et al. 2005 for a similar discussion about the HZ around red giants). The second implication is that habitable planets were initially on the hot side of the HZ. Around a Sun-like star such a hot location would imply atmospheric losses of water (Lammer et al. 2010). Venus, for instance, has kept little of its initial water reservoir, as shown by the high $\mathrm{D} / \mathrm{H}$ ratio of its remaining water (a few tens of $\mathrm{cm}$ precipitable). If planets lose most of their water content during their pre-habitable history, they are unlikely to become habitable worlds when the HZ catches up with them. The case of Venus, however, may not be a relevant analog for BD planets. Indeed, the Sun emits significant UV and XUV fluxes, respectively able to photolyze $\mathrm{H}_{2} \mathrm{O}$ and to drive the atmospheric escape by heating the exosphere.

It is unclear whether BDs have enough activity to produce such fluxes that would result in a significant water loss. For $\mathrm{G}, \mathrm{K}$, and early-M stars, magnetic activity and resulting XUV emission is correlated with rotation rate (Ribas et al. 2005; Scalo et al. 2007) and thus the XUV levels in the HZ of early-M stars remain very high (higher than in the $\mathrm{HZ}$ of the Sun) for 1 to a few Gyr. For late-M stars and BDs, and despite their high rotation rate, there is a steep drop-off of activity, which may be explained by their lower atmospheric temperature and ionization fraction (Mohanty et al. 2002). Very young BDs do exhibit observable X-rays (Preibisch et al. 2005) but these are likely to come from the accretion of a protoplanetary disk ergo predating the formation of planets. Therefore in the absence of significant photolysis and exospheric heating, it is possible for planets on the hot side of the HZ of a BD to keep a steam atmosphere long enough to become habitable. Note also that, even in the case of significant atmospheric and water erosion, the amount of water that remains for the habitability window depends on the initial reservoir. Volatile-rich planets, or so-called ocean planets, that have formed in the cold outer part of the protoplanetary disk and migrated toward inner regions, can keep more than a terrestrial ocean for billions of years even if located close to a Sun-like star (Selsis et al. 2007a). It is therefore possible to have oceans at the surface of planets in the HZ of BDs.

Since the submission of this article, Barnes \& Heller (2013) have independently addressed and quantitatively furthered some of the questions raised above in this section; specifically, they include the potential effect of tidal heating when the planet eccentricity is forced to non-zero values by planet-planet interactions.

\subsection{Biosignatures from BD Planets}

The atmospheric biosignatures paradigm rests on the ability to detect an out-of-equilibrium thermodynamical state and that all simpler physical processes fail to reproduce this state. For instance, the photolysis and escape mentioned above can lead to abiotic $\mathrm{O}_{2}$ buildup. Unfortunately a back of the envelope calculation shows that a detectable level of UV emission from nearby BDs (with the HST) corresponds to levels in their habitable zone significantly higher than the ones required for such $\mathrm{O}_{2}$ build-up.

Supposing that all simpler physical causes for the observed out-of-equilibrium state are ruled out it is then likely that a more complex process is responsible. On Earth it is oxygenic photosynthesis (and not chemoautotrophy) that is responsible for the out-of-equilibrium state of our atmosphere (Rosing 2005). Oxygenic photosynthesis uses photons to break water molecules and the liberated hydrogen to reduce $\mathrm{CO}_{2}$. The water bonding energy corresponds to a wavelength of $240 \mathrm{~nm}$. As no such short wavelength reaches the surface of the Earth life has adapted to the available, longer wavelength spectrum and eventually managed to store the energy of the number of photons required for breaking a single $\mathrm{H}_{2} \mathrm{O}$ molecule. Using the even longer wavelengths of a BD may imply only the ability to store more photons per $\mathrm{H}_{2} \mathrm{O}$ molecule. Actually, photosynthesis may have evolved from initial infrared sensors used to detect sources of heat (Nisbet et al. 1995), and there are claims that photosynthetic organisms still use infrared light at great oceanic depth (Beatty et al. 2005). There is, therefore, no reason to rule out the possibility of photosynthetic activity (oxygenic or not) evolving on a BD planet.

So going back to BDs possibly lacking a photochemical out-of-equilibrium influence on their HZ planets, detection of biosignatures would be a most robust detection of life.

The question of how to detect $\mathrm{O}_{2}$ remains. BDs do not provide enough flux at $760 \mathrm{~nm}$ for primary eclipse spectroscopy detection (except perhaps for the brightest BDs). Also no $\mathrm{O}_{3}$ build-up is expected (Section 5). Therefore, detecting $\mathrm{O}_{2}$ in the absence of significant photochemistry and visible flux 
seems to be challenging. One path could be to investigate the observability of the dimers $\mathrm{O}_{2}-\mathrm{X}_{2}\left(\mathrm{O}_{2}-\mathrm{O}_{2}, \mathrm{O}_{2}-\mathrm{N}_{2}\right.$, etc. $)$ at $1.26 \mu \mathrm{m}$, in the extended column at the limb during primary transits (Pallé et al. 2009, Figure S3).

\section{CONCLUSION}

In this paper, we have examined three different strategies for detecting eclipsing habitable planets around BDs, depending on the maximum habitable orbital period. These group into ground searches and into space-based searches (Spitzer Space Telescope).

Planets orbiting massive and old BDs can have a maximum habitable orbital period shorter than the duration of the observing night. Though they may be rare they can be screened for with $100 \%$ completeness in only one night, from a single location (one telescope). Conducting a transit search in the near-infrared is mandatory.

We have started investigating the monitoring of bright BDs with the $2 \mathrm{~m}$ class telescope network (multiple institutions) in the deep red optical. Coordinated observations with a longitudedistributed network of telescopes can increase the duration of the continuous monitoring sequences therefore reducing the time cost for achieving satisfactory screening completeness for a given target. However, weather statistics at the various sites (and their correlation) should be included in estimating the time cost of such campaigns, and the need for additional longituderedundant coordination. It is the third option-monitoring from space-which appears to be the most effective.

We also show that the density of habitable eclipsing planets around BDs varies greatly depending on mechanisms that have only recently started to be investigated around BDs. Consider a survey of the habitable orbits for the 21 closest BDs ( $7 \mathrm{pc}$ ). The likelihood of detecting at least one (habitable) transiting planet varies between $4.5_{-1.4}^{+5.6} \%$ and $56_{-13}^{+31} \%$, depending on whether tidal evolution is taken into account or not. Even in the pessimistic case, since these planets are also remarkably easy to find if they do exist, a search program is worth the risk since it can validate these newly investigated mechanisms (tidal-induced migration).

Occultation spectroscopic characterization of a habitable planet around a BD within $5-10 \mathrm{pc}$ is achievable with the $J W S T$. Such a program would be spread over only one-fifth to one-tenth of the mission's lifetime (instead of the whole mission for planets around $\mathrm{M}$ dwarfs).

More generally, given uncertainties in the existence of available nearby eclipse-characterizable habitable planets, an effort should be made to upgrade transit surveys around low-mass dwarf projects to increased sensitivity (as planned in the recent Berta et al. 2012, but also increased collector diameter, better filters, and better sites). Setting up new surveys is also to be considered. The completeness of the screening for eclipsing habitable planets around nearby low-mass dwarfs should be published at the earliest to enable, if required, the adjustment of roadmaps toward their characterization in combined light. In the case of the absence of eclipsing planets, the effort toward characterization should once again be fully redirected to the spatially resolving track mentioned in the Introduction. ${ }^{20}$ The interest of a local sample is not only in terms of astronomical

\footnotetext{
20 Combined light spectroscopic characterization of non-eclipsing habitable super-Earths may be achievable with the JWST for extremely favorable (e.g., among other, most nearby) cases - see preliminary work by Selsis et al.

(2011). Completeness of the screening for this population may be achieved by a mission like the NEAT proposal (Malbet et al. 2011).
}

photon $\mathrm{S} / \mathrm{N}$ but also inspirational for future generations (e.g., probe sending; see also Belu 2011).

We are most thankful to Amaury Triaud, Michael Gillon, Roi Alonso, and Monika Lendl for helping with this work. A.B. acknowledges support from CNES. F.S. acknowledges support from the European Research Council (ERC) starting grant 209622: $E_{3} A_{R T H}{ }^{s}$. SNR thanks the CNRS's PNP program. P.F. acknowledges support from the ERC/European Community under the FP7 through Starting Grant agreement number 239953, as well as by Fundação para a Ciência e a Tecnologia (FCT) in the form of grant reference PTDC/CTE-AST/098528/2008. This research made use of www.solstation.com, Aladin, and JSkyCalc. A.B. thanks J.-F. Lecampion for assistance with light curve production. We thank Ludovic Puig for contributing to the inspiration for this work. We thank the anonymous referee for attentive reading that helped improve the manuscript and the numerous educational and prospective comments.

\section{Facilities: FTN, FTS, HCT}

\section{REFERENCES}

Abbot, D. S., \& Switzer, E. R. 2011, ApJL, 735, L27

Andreeschev, A., \& Scalo, J. 2004, in IAU Symp. 213, in Bioastronomy 2002: Life among the Stars, ed. R. Norris \& F. Stootman (San Francisco, CA: ASP), 115

Andrews, S. M., Wilner, D. J., Hughes, A. M., Qi, C., \& Dullemond, C. P. 2010, ApJ, 723, 1241

Apai, D., Pascucci, I., Bouwman, J., et al. 2005, Sci, 310, 834

Artigau, E., Bouchard, S., Doyon, R., \& Lafrenière, D. 2009, ApJ, 701, 1534

Artigau, E., Radigan, J., Folkes, S., et al. 2010, ApJL, 718, L38

Baraffe, I., Chabrier, G., Barman, T. S., Allard, F., \& Hauschildt, P. H. 2003, A\&A, 402, 701

Barnes, R., \& Heller, R. 2013, AsBio, in press (arXiv:1211.6467)

Beatty, J. T., Overmann, J., Lince, M. T., et al. 2005, PNAS, 102, 9306

Beaulieu, P., Kipping, D. M., \& Batista, V. 2010, MNRAS, 409, 963

Beckwith, S. V. W. 2008, ApJ, 684, 1404

Beckwith, S. V. W., Stiavelli, M., Koekemoer, K., et al. 2006, AJ, 132, 1729

Belu, A. R. 2011, in Studies in Space Policy, Vol. 5, ESPI \& ESF, ed. U.

Landfester, N.-L. Remuss, K.-U. Schrogl, \& J.-C. Worms (Berlin: Springer), 57

Belu, A. R., Selsis, F., Morales, J.-C., et al. 2011, A\&A, 525A, 83

Berta, Z., Irwin, J., Charbonneau, D., Burke, C., \& Falco, E. E. 2012, AJ, 144,145

Blake, C. H., Bloom, J. S., Latham, D. W., et al. 2008, PASP, 120, 860

Blake, C. H., \& Shaw, M. M. 2011, PASP, 123, 1302

Bolmont, E., Raymond, S. N., \& Leconte, J. 2011, A\&A, 535A, 94

Bonfils, X., Delfosse, X., Udry, S., et al. 2013, A\&A, 549, A109

Burgasser, A. J., Tinney, C. G., Cushing, M. C., et al. 2008, ApJL, 689, L53

Burningham, B., Leggett, S. K., Lucas, P. W., et al. 2010, MNRAS, 404, 1952

Caballero, J. A. 2010, Highlights of Spanish Astrophysics V, Astrophysics and Space Science Proceedings (Berlin: Springer), 79

Caballero, J. A., \& Rebolo, R. 2002, in First Eddington Workshop on Stellar Structure and Habitable Planet Finding, ed. F. Favata, I. W. Roxburgh, \& D. Galadí Enríquez (ESA SP-485; Noordwijk: ESA), 261

Charbonneau, D., Irwin, J., Nutzman, P., \& Falco, E. E. 2008, BAAS, 40, 242

Charnoz, S., Salmon, J., \& Crida, A. 2010, Natur, 465, 752

Cockell, C. S., Léger, A., Fridlund, M., et al. 2009, AsBio, 9, 1

Cushing, M. C., Kirkpatrick, J. D., Gelino, C. R., et al. 2011, ApJ, 743, 50

Del Burgo, C., Martín, E. L., Zapatero Osorio, M. R., \& Hauschildt, P. H. 2009, A\&A, 501, 1059

Deming, D. L., Seager, S., Winn, J., et al. 2009, PASP, 121, 952

Eggl, S., Pilat-Lohinger, E., Georgakarakos, N., Gyergyovits, M., \& Funk, B. 2012, ApJ, 752, 74

Faber, J. A., Rasio, F. A., \& Willems, B. 2005, Icar, 175, 248

Faherty, J. K., Burgasser, A. J., Cruz, K. L., et al. 2009, AJ, 137, 1

Ford, E. B., \& Rasio, F. A. 2006, ApJL, 638, L45

Geißler, K., Chauvin, G., \& Sterzik, M. F. 2008, A\&A, 480, 193

Grillmair, C. J., Burrows, A., Charbonneau, D., et al. 2008, Natur, 456, 767

Herbst, W., Eislöffel, J., Mundt, R., \& Scholz, A. 2007, in Protostars \& Planets V, ed. B. Reipurth, D. Jewitt, \& K. Keil (Tucson, AZ: Univ. Arizona Press), 297 
Holman, M. J., \& Wiegert, P. A. 1999, AJ, 117, 621

Jackson, B., Barnes, R., \& Greenberg, R. 2008, MNRAS, 391, 237

Jayawardhana, R., Mohanty, S., \& Basri, G. 2003, ApJ, 592, 282

Joshi, M. 2003, AsBio, 3, 415

Kaltenegger, L., \& Traub, W. A. 2009, ApJ, 698, 519

Kasper, M., Biller, B. A., Burrows, A., et al. 2007, A\&A, 471, 655

King, R. R., McCaughrean, M. J., Homeier, D., et al. 2010, A\&A, 510A, 99

Kirkpatrick, J., Cushing, M. C., Gelino, C. R., et al. 2011, ApJS, 197, 19

Knapp, G. R., Leggett, S. K., Fan, X., et al. 2004, AJ, 127, 3553

Lammer, H., Selsis, F., Chassefière, E., et al. 2010, AsBio, 10, 45

Leconte, J., Lai, D., \& Chabrier, G. 2011, A\&A, 528A, 41

Leggett, S. K., Burningham, B., Saumon, D., et al. 2010, ApJ, 710, 1627

Leggett, S. K., Cushing, M. C., Saumon, D., et al. 2009, ApJ, 695, 1517

Leggett, S. K., Saumon, D., Marley, M. S., et al. 2012, ApJ, 748, 74

Lopez, B., Schneider, J., \& Danchi, W. C. 2005, ApJ, 627, 974

Luhman, K. L., Adame, L., D’Alessio, P., et al. 2005, ApJL, 635, L93

Malbet, F., Léger, A., Shao, M., et al. 2011, ExA Online First, 109

Mohanty, S., Basri, G., Shu, F., Allard, F., \& Chabrier, G. 2002, ApJ, 571, 469

Nisbet, E. G., Cann, J. R., \& Van Dover, C. L. 1995, Natur, 373, 479

Paczyński, B. 1971, ARA\&A, 9, 183

Pallé, E., Zapatero Osorio, M. R., \& García Muñoz, A. 2011, ApJ, 728, 19

Pallé, E., Zapatero Osorio, M. R., \& Barrena, R. 2009, Natur, 459, 814

Payne, M. J., \& Lodato, G. 2007, MNRAS, 381, 1597
Preibisch, T., McCaughrean, M. J., Grosso, N., et al. 2005, ApJS, 160, 582

Rauer, H., Gebauer, S., Paris, P. V., et al. 2011, A\&A, 529A, 8

Raymond, S. N., Scalo, J., \& Meadows, V. S. 2007, ApJ, 669, 606

Ribas, I., Guinan, E. F., Güudel, M., \& Audard, M. 2005, ApJ, 622, 680

Rosing, M. T. 2005, IJAsB, 4, 9

Saunders, E. S., Naylor, T., \& Allan, A. 2008, AN, 329, 321

Scalo, J., Kaltenegger, L., Segura, A., et al. 2007, AsBio, 7, 85

Scholz, R.-D., Bihain, G., Schnurr, O., \& Storm, J. 2011, A\&A, 532L, 5

Scholz, R.-D., Storm, J., Knapp, G. R., \& Zinnecker, H. 2009, A\&A, 494, 949

Selsis, F., Chazelas, B., Bordé, P., et al. 2007a, Icar, 191, 453

Selsis, F., Kasting, J. F., Levrard, B., et al. 2007b, A\&A, 476, 1373

Selsis, F., Wordsworth, R. D., \& Forget, F. 2011, A\&A, 532A, 1

Stephens, D. C., Leggett, S. K., Cushing, M. C., et al. 2009, ApJ, 702, 154

Stevenson, K. B., Harrington, J., Nymeyer, S., et al. 2010, Natur, 464, 1161

Struve, O. 1952, Obs, 72, 199

Swain, M. R., Vasisht, G., Tinetti, G., et al. 2009, ApJL, 690, L114

Tinetti, G., Vidal-Madjar, A., Liang, M.-C., et al. 2007, Natur, 448, 169

Traub, W., Shaklan, S., \& Lawson, P. 2007, in Proc., In the Spirit of Bernard

Lyot: The Direct Detection of Planets and Cirumstellar Disks in the 21st

Century, ed. P. Kalas (Berkely, CA: Univ. California Berkeley), 36

Volk, K., Blum, R., Walker, G., \& Puxley, P. 2003, IAU Circ., 8188, 2

von Braun, K., Kane, S. R., Ciardi, D. R., et al. 2009, ApJ, 702, 779

Wordsworth, R., Forget, F., Selsis, F., et al. 2011, ApJL, 733, L48 